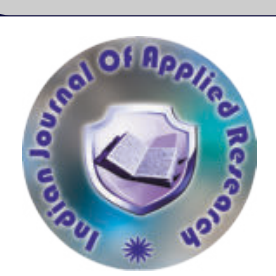

Radiology

AGREEMENT BETWEEN MULTIDETECTOR COMPUTED TOMOGRAPHY

AND ENDOSCOPY FOR THE EVALUATION OF GASTRO-ESOPHAGEAL VARICES IN PATIENTS WITH PORTAL HYPERTENSION.

\begin{tabular}{l} 
Mehak Garg* \\
\hline $\begin{array}{l}\text { Nirmal Kumar } \\
\text { Prabhu }\end{array}$
\end{tabular}

Shivane Garg

Saravanan S

\section{Rubalakshmi S}

Srikanth Moorthy
Department of Radiodiagnosis, Amrita Institute of Medical Sciences, Amrita School Medicine, Amrita Vishwa Vidyapeetham, Cochin, Kerala, India. *Corresponding Author Department of Radiodiagnosis, Amrita Institute of Medical Sciences, Amrita School Medicine, Amrita Vishwa Vidyapeetham, Cochin, Kerala, India. Department of Radiodiagnosis, All India Institute of Medical Sciences, Bathinda, Punjab, India.

Department of Radiodiagnosis, Amrita Institute of Medical Sciences, Amrita School Medicine, Amrita Vishwa Vidyapeetham, Cochin, Kerala, India.

Department of Radiodiagnosis, Amrita Institute of Medical Sciences, Amrita School Medicine, Amrita Vishwa Vidyapeetham, Cochin, Kerala, India.

Department of Radiodiagnosis, Amrita Institute of Medical Sciences, Amrita School Medicine, Amrita Vishwa Vidyapeetham, Cochin, Kerala, India.

ABSTRACT AIM: The aim is to study the agreement between MDCT and endoscopy in diagnosing gastroesophageal varices in patients of chronic liver disease with portal hypertension, keeping endoscopy as gold standard. MATERIALS AND METHODS: The study included 29 cirrhotic patients with portal hypertension assessed between August 2018 to August 2020 who underwent upper gastrointestinal (GI) endoscopy and MDCT within 12 weeks. Two radiologists reviewed the scans, to determine the grade of esophageal varices. To evaluate the accuracy of MDCT findings by Radiologists 1 and 2 with respect to endoscopy findings (GOLD STANDARD), McNemar's chi square test with validity parameters such as Sensitivity, Specificity, Positive predictive value, Negative predictive value and accuracy was computed. Cohens kappa was used to ascertain the inter-observer agreement for grading of varices between MDCT and endoscopy. RESULTS: The sensitivity of MDCT for radiologist A was $76.7 \%$, specificity $93 \%$, Accuracy $89.7 \%$, PPV 91.6\%, NPV 93.9\% and for radiologist B, $79 \%, 93.4 \%, 89.6 \%, 81.4 \%$ and $93.1 \%$ respectively. For the inter-rater agreement the kappa value between Radiologist A v/s upper GI endoscopy was 0.716 , Radiologist B v/s upper GI endoscopy was 0.720 and Radiologist A v/s Radiologist B was 0.808 . MDCT detected para esophageal varices in 16 cases, gastric fundus varices in 9 cases and splenorenal collaterals in 3 cases, palisade vein dilatation was positive in 9 cases, negative in 20 cases. 3 cases of HCC were incidentally found during examination. CONCLUSION: MDCT is a good replacement for endoscopy in the detection of gastroesophageal varices, especially those with high grade varices (grade 2 and 3). MDCT helps in detection of palisade vein and other extra-luminal findings like HCC, other portosystemic collaterals.

KEYWORDS : Cirrhosis, Endoscopy, Computed Tomography, Portal hypertension.

\section{INTRODUCTION:}

Portal hypertension is defined as a hepatic venous pressure gradient (HVPG) above $5 \mathrm{mmHg}$ and is a serious complication of cirrhosis. When the hepatic venous pressure gradient increases above $10 \mathrm{mmHg}$, it leads to the development of complications of portal hypertension in the form of ascites and/or esophageal and gastric varices $(1,2)$. Gastroesophageal varices are the most recognized portosystemic collaterals because their rupture results in dangerous variceal bleeding, which is considered as the commonest lethal complication of portal hypertension (3). Esophagogastroduodenoscopy (EGD) is the gold standard in the diagnosis of gastroesophageal varices; however, the use of endoscopy as a method of screening is limited because it is invasive, expensive, needs sedation and there is poor acceptance of the procedure by patience $(4,5)$. To limit the number of patients who undergo endoscopic screening, a noninvasive, less expensive and well tolerated test for diagnosis of varices with high sensitivity and specificity is needed. Computed tomography (CT) imaging is noninvasive, does not necessitate sedation, and allows accurate assessment of variceal site and size, and it is also better tolerated by most of the patients than endoscopy. With rapid evolution of CT technology, especially the introduction of multi-detector computed tomography (MDCT) imaging with its multiplanar capabilities, esophageal, paraoesophageal and gastric varices as well as other portosystemic shunts can be progressively recognized in patients with liver cirrhosis $(6,7)$. The primary objective of the study was to study the agreement between MDCT and endoscopy in diagnosing gastroesophageal varices in patients of chronic liver disease with portal hypertension, keeping endoscopy as gold standard.

\section{MATERIALS AND METHODS:}

Institutional Review Board approval was taken for this prospective cross-sectional study. Informed consent was taken from all the patients before they underwent CT. Data set consists of all patients with liver cirrhosis who underwent triple phase CT from the department of
Radio-Diagnosis of Amrita Institute of Medical Sciences research Centre, Kochi and Endoscopy from the Department of Gastromedicine of Amrita Institute of Medical Sciences research Centre, Kochi during the period of 2018-2020. MDCT was done first followed by Endoscopy. A total of 29 patients with liver cirrhosis were included in study. All the patients with chronic liver disease and portal hypertension who were willing to undergo MDCT and Endoscopic evaluation were included in the study. The patients with active gastrointestinal hemorrhage, history of endoscopic variceal ligation, history of adverse reactions to iodinated contrast agent, with known congenital anomalies of the portal vein and patients who refused to do endoscopy after CT angiography were excluded.

All CT examinations were performed using 256 slice MDCT (Brilliance-iCT;Philips Healthcare, Cleveland, $\mathrm{OH}$ ) after intravenous injection of non-ionic iodinated contrast agent, Iohexol (Omnipaque ${ }^{\mathrm{TM}}$ 350, GE Healthcare, Princeton, NJ) over a period of 25 seconds. A $1.5 \mathrm{~mL} / \mathrm{Kg}$ body weight of the contrast was administered to the patients. The contrast was injected using power injector (OptiVantage, Guerbet, $\mathrm{OH}$ ). Bolus triggering was used to obtain the contrast enhanced phases. The region of interest was placed over descending thoracic aorta, $2 \mathrm{~cm}$ proximal to the diaphragm and scanning was initiated after the threshold of $100 \mathrm{HU}$ was reached. Arterial dominant phase images were acquired at 25 seconds, portal dominant phase images were acquired at 45 seconds and delayed hepatic venous phase images were acquired at 100 seconds after the injection. All scans will be performed utilizing the high-quality scan mode, at $1.25-\mathrm{mm}$ slice thickness, and reconstruction Intervals of $0.625 \mathrm{~mm}$ for portal venous phase imaging. Images were transferred to a workstation and multiplanar reformation (MPR) images were obtained in coronal and sagittal sections at 1-mm thickness in the region where varices will be detected. The portal dominant phase images of enhanced CT images was used for evaluation of the esophageal varices in detail. All CT 
images were interpreted by two independent radiologists (A and $\mathrm{B}$ ). On MDCT images the following data was taken into consideration: Number of varices, size and diameter of varices and then Grading of esophageal varices was done by MDCT according to classification proposed by Shimizu et al. (8). Grade 0: no varices visualization on the inner surface of the esophagus, Grade 1: one varix less than $5 \mathrm{~mm}$ in diameter detected on inner surface from the esophagus, Grade 2: several varices less than $5 \mathrm{~mm}$ detected on the inner surface from the esophagus, and Grade 3: one varix $5 \mathrm{~mm}$ or greater or several varices occupy more than half of the circumference of the esophagus. All the patients underwent Upper GIT endoscopy within 2 weeks following CT study; for the grading of varices and presence or absence of RC sign.

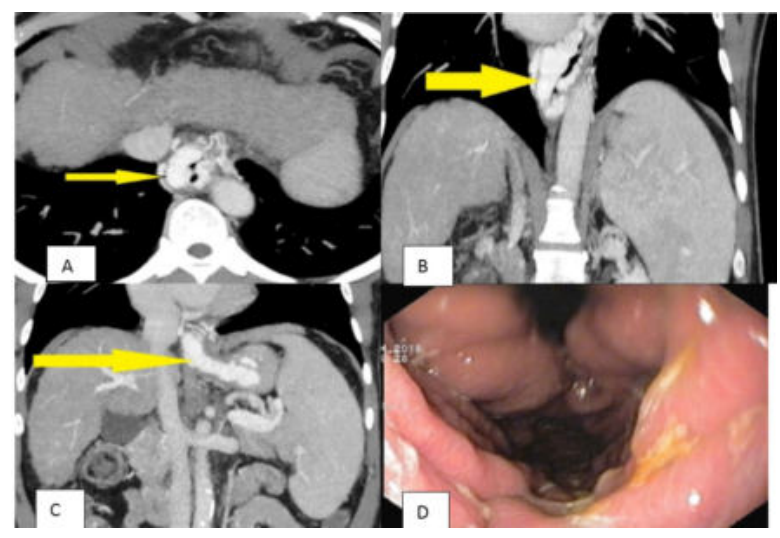

Figure 1:

A 66-years-old male patient with cirrhosis and portal hypertension and esophageal varices. (a) CT axial reformat post-contrast portal venous phase image shows enhanced intraluminal esophageal varices involving almost the whole circumference of the inner surface of lower esophagus with large varix measuring $9 \mathrm{~mm}$ in diameter (yellow arrow) (Grade 3). (b) CT saggital reformat shows enhanced vascular structures in the esophageal lumen (yellow arrow). (c) CT coronal reformat images show a large palisade vein coursing from the lower esophagus down to the gastric region with diameter of $9 \mathrm{~mm}$. (d) Upper GIT endoscopy shows multiple lobulated submucosal esophageal varices (grade 3 (CT Score 3, endoscopy grade 3, RC 0).

\section{STATISTICALANALYSIS:}

Statistical analysis was done using IBM SPSS 20. (SPSS Inc, Chicago, USA). To test the statistical significance of the difference in the association between the categorical variable Chi square test with fishers exact test was used. To find out the accuracy of MDCT findings of Radiologists 1 and 2 with respect to endoscopy findings, McNemar's chi square test with validity parameters such as Sensitivity, Specificity, Positive predictive value, Negative predictive value and accuracy was computed. The inter-observer agreement for grading of varices between MDCT and endoscopy are studied by Cohens Kappa. A Pvalue of $<0.05$ was considered as statistically significant

\section{RESULTS:}

The mean age of patients was 60.48 \pm 9.72 years (Male: Female: 24:5). MDCT compared to endoscopy with endoscopy as gold standard for the detection and grading of esophageal varices showed a sensitivity $76.7 \%$, specificity $93 \%$, PPV91.6\%, NPV $93.9 \%$ and accuracy $89.7 \%$ according to Radiologist A and a sensitivity of $79 \%$, specificity $93.4 \%$, PPV81.4\%, NPV93.1\% and accuracy $89.6 \%$ according to Radiologist $\mathrm{B}$. The inter-rater agreement between the MDCT read by Radiologist A and upper GI endoscopy was substantial agreement with a kappa value of 0.716 , between Radiologist B and upper GI endoscopy was also substantial agreement with a kappa value of 0.720 , and between Radiologist A and B there was a perfect agreement with a kappa value of 0.808 (Table 1). The accuracy for detection of high grade (i.e, grade 2 and 3) by both the Radiologists was higher as compared to low grade varices (grade 0 or 1) ( Figure 2). MDCT also detected various extraluminal findings like para esophageal varices in $16(55.2 \%)$ cases, gastric fundus varices in 9 cases $(26 \%)$ and splenorenal collaterals were seen in 3 cases $(10.3 \%)$, palisade vein dilatation was $+v e$ in $9(31.1 \%)$ cases, -ve in $20(68.9 \%)$ cases. 3 cases $910.3 \%)$ of HCC were incidentally found during examination.
Table 1: Inter-rater agreement between radiologist $A$ and $B$ and endoscopy regarding detection and grading of esophageal varices.

\begin{tabular}{|l|l|l|}
\hline \multirow{2}{*}{ Observers } & \multicolumn{2}{|l|}{ Inter-rater agreement } \\
\cline { 2 - 3 } & Kappa & p-value \\
\hline Radiologist A versus upper GI endoscopy & 0.716 & 1.000 \\
\hline Radiologist B versus upper GI endoscopy & 0.720 & 1.000 \\
\hline Radiologist A versus Radiologist B & 0.808 & 1.000 \\
\hline
\end{tabular}

Figure 2: Comparison of the accuracy of Radiologist $A$ and $B$ in detecting the grades of varices, keeping endoscopy as gold standard.

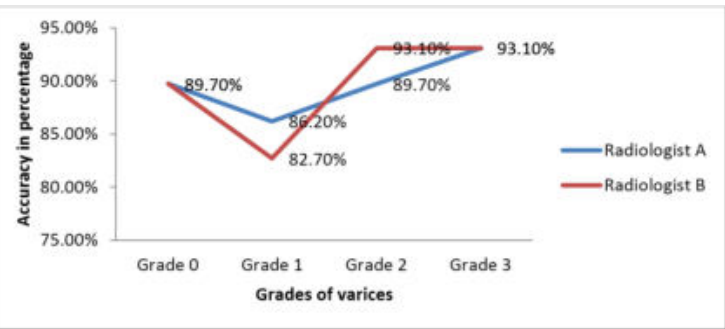

\section{DISCUSSION}

Variceal bleeding is a serious adverse event in patients with liver cirrhosis. Patients survive the 1 st episodes of variceal bleeding have a greater than $60 \%$ risk of recurrent hemorrhage within 1 st year of recurrent episode (9). Out of the evaluated patients ,24(82.8\%) were males and 5(17.2\%) were females. The study group had age distribution between 40 and 80 years, with mean age $60.48 \pm 9.01$ years. MDCT findings were read by two different radiologist-Radiologist A and $\mathrm{B}$, one with 25 years of experience and other with about 10 years of experience in radiology. Both the radiologists were blinded to the results and the grading given by the endoscopy for esophageal varices. Once the readings were done by both the radiologists, the results were compared with those of endoscopy results and with each other. The difference between radiologists A and B in detecting different grades of esophageal varices was insignificant. Also, there was a good agreement between radiologists A, B and upper GIT endoscopy regarding detection and grading of esophageal varices. This agreement study was similar to the article by Tarek ELKammmash (10) et al to evaluate the role of multidetector computed tomography (MDCT) in evaluation of esophageal varices (EV). In examining presence or absence of palisade vein dilatation, we found that palisade vein is present in 10 of the cases and absent in 19 cases. On correlation of these negative cases with the grade of varices, we found these cases either do not have varices or low grade varices. In contrary, we found that cases which were evaluated as positive for presence of palisade vein tend to have higher grade varices. Also, we observed that there is increase in the degree of vein dilatation with increasing grades of $\mathrm{EV}(\mathrm{p}<0.01)$. These highly significant correlation results were in agreement with those of Dessouky and Abdel Aal. (11). A prominent conclusion made in our study was that the agreement between the endoscopy and MDCT for the detection of high-grade varices (grade II and III combined) is very good(kappa value $>0.81$ ). The high-grade varices are also clinically significant, being associated with a high risk of upper GI bleed. Due to the high degree of agreement, MDCT follow-up of these varices is therefore adequate and invasive endoscopy can be avoided purely for screening purposes. Regarding the incidental detection of other portosystemic collaterals, in our study we were able to detect the presence of paraesophageal varices in 16 cases, gastric fundus varices in 9 cases, whereas splenorenal collaterals were seen in 3 cases. The advantage of high speed multidetector CT is that it was able to identify them and differentiate them from esophageal varices, while on endoscopy only esophageal varices can be visualized and this was in agreement with Kodama et al. (6) and Mifune et al. (12) who clarified the important advantage of multi-detector row CT over single-detector row helical CT and conventional portography is the increased speed of scanning, which permits routine use of very thin collimation for imaging the portosystemic collateral vessels whereas collateral vessels can now be demonstrated without the risk, discomfort and invasiveness of catheterization. In addition to these other portosystemic shunts, MDCT was able to diagnose 3 cases of HCC within cirrhotic liver patients and differentiate those from another case of simple hepatic cyst during its routine protocol scanning for esophageal varices and this gives other advantage of MDCT over endoscopy and other invasive procedures. And this was in agreement with Kim et al. (13), who stated that considering the high cost of 
performing multiple tests and the relative invasiveness of endoscopy, a single noninvasive surveillance tool for both varices and HCC may be important.

\section{CONCLUSION:}

MDCT is a good replacement of endoscopy for the evaluation of gastroesophageal varices, especially for high grade varices. MDCT can reliably detect high grade varices (grade 2 and 3) with a high degree of agreement with endoscopy. Patients with low grade varices (grade 0 and 1) can be followed up on MDCT (after an initial endoscopy confirmation)-in order to detect progression to grade $2 / 3$. MDCT helps in detection of other extra-luminal findings like HCC, other portosystemic collaterals, which can be a useful guide to plan BORTO procedure.

\section{REFERENCES:}

1. Biecker, E. (2013). Gastrointestinal Bleeding in Cirrhotic Patients with Portal Hypertension. ISRN Hepatology, 2013, 1-20. https://doi.org/10.1155/2013/541836

2. Groszmann, R. J., Garcia-Tsao, G., Bosch, J., Grace, N. D., Burroughs, A. K., Planas, R., Escorsell, A., Garcia-Pagan, J. C., Patch, D., Matloff, D. S., Gao, H., \& Makuch, R. (2005). Beta-Blockers to Prevent Gastroesophageal Varices in Patients with Cirrhosis. New England Journal of Medicine, 353(21), 2254-2261. https://doi.org/10.1056/ NEJMoa044456

3. Garcia-Tsao, G., Sanyal, A. J., Grace, N. D., Carey, W., \& Practice Guidelines Committee of the American Association for the Study of Liver Diseases, the Practice Parameters Committee of the American College of Gastroenterology. (2007). Prevention and management of gastroesophageal varices and variceal hemorrhage in cirrhosis. Hepatology, 46(3), 922-938. https://doi.org/10.1002/hep.21907

4. Eisen, G. M., Eliakim, R., Zaman, A., Schwartz, J., Faigel, D., Rondonotti, E., Villa, F., Weizman, E., Yassin, K., \& deFranchis, R. (2006). The Accuracy of PillCam ESO Capsule Endoscopy Versus Conventional Upper Endoscopy for the Diagnosis of Esophageal Varices: A Prospective Three-Center Pilot Study. Endoscopy, 38(01), 31-35. https://doi.org/10.1055/s-2005-921189

5. Terayama, N., Matsui, O., Kobayashi, S., Sanada, J., Gabata, T., Koda, W., \& Minami, T. (2008). Portosystemic shunt on CT during arterial portography: Prevalence in patients with and without liver cirrhosis. Abdominal Imaging, 33(1), 80-86. https://doi.org/10. 1007/s00261-007-9196-2

6. Kodama, H., Aikata, H., Takaki, S., Azakami, T., Katamura, Y., Kawaoka, T., Hiramatsu, A., Waki, K., Imamura, M., Kawakami, Y, Takahashi, S., Toyota, N., Ito, K., \& Chayama, K. (2010). Evaluation of portosystemic collaterals by MDCT-MPR imaging Chayama, K. (2010). Evaluation of portosystemic collaterals by MDCT-MPR imaging
for management of hemorrhagic esophageal varices. European Journal of Radiology, 76(2),239-245. https://doi.org/10.1016/j.ejrad.2009.06.011

7. Kang, H. K., Jeong, Y. Y., Choi, J. H., Choi, S., Chung, T. W., Seo, J. J., Kim, J. K., Yoon, W., \& Park, J. G. (2002). Three-dimensional Multi-Detector Row CT Portal Venography in the Evaluation of Portosystemic Collateral Vessels in Liver Cirrhosis. RadioGraphics, 22(5), 1053-1061. https://doi.org/10.1148/radiographics.22.5. g02se011053

8. Shimizu, T., Namba, R., Matsuoka, T., Tabuchi, K., Yamamoto, K., Uesugi, Y., Matsui, R., Sueyoshi, K., \& Narabayashi, I. (1999). Esophageal varices before and after R., Sueyoshi, K., \& Narabayashi, I. (1999). Esophageal varices before and after
endoscopic variceal ligation: Evaluation using helical CT. European Radiology, 9(8), endoscopic variceal ligation: Evaluation using helical

9. de Franchis, R. (2015). Expanding consensus in portal hypertension. Journal of Hepatology, 63(3), 743-752. https://doi.org/10.1016/j.jhep.2015.05.022

10. ELKammash, T., ELFiky, I., Zaiton, F., \& Khorshed, S. E. (2016). Diagnostic performance of multidetector computed tomography in the evaluation of esophageal varices. The Egyptian Journal of Radiology and Nuclear Medicine, 47(1), 43-51. https://doi.org/10.1016/j.ejrnm.2015.11.003

11. Dessouky, B. A. M., \& Abdel Aal, E. S. M. (2013). Multidetector CT oesophagography: An alternative screening method for endoscopic diagnosis of oesophageal varices and An alternative screening method for endoscopic diagnosis of oesophageal varices and
bleeding risk. Arab Journal of Gastroenterology, 14(3), 99-108. https://doi.org/10. 1016/j.ajg.2013.08.006

12. Mifune, H., Akaki, S., Ida, K., Sei, T., Kanazawa, S., \& Okada, H. (2007). Evaluation of esophageal varices by multidetector-row CT: Correlation with endoscopic "red color sign." Acta Medica Okayama, 61(5), 247-254. https://doi.org/10.18926/AMO/32894

13. Kim, S. H., Han, J. K., Lee, K. H., Chung, J. W., Yang, H. K., \& Choi, B. I. (2003). Computed Tomography Gastrography With Volume-rendering Technique: Correlation With Double-contrast Barium Study and Conventional Gastroscopy: Journal of Computer Assisted Tomography, 27(2), 140-149. https://doi.org/10.1097/00004728200303000-00006. 